\title{
Spectral Behavior and Photophysical Parameters of Dihydrophenanthro[9,10-e][1,2,4]Triazine Derivative Dyes in Sol-gel and Methyl Methacrylate Polymer Matrices
}

\author{
Maram T. H. Abou Kana \\ Cairo University \\ Ahmed H.M. Elwahy \\ Cairo University

\section{Samy A. El-Daly A. El-Daly} \\ Tanta University \\ El-Zeiny M. Ebeid \\ Tanta University
}

Mahmoud Sakr ( $\nabla$ M.sakr@niles.edu.eg )

Cairo University National Institute of Laser Enhanced Sciences https://orcid.org/0000-0003-3578-6157

\section{Research Article}

Keywords: Optical property, Solid state laser dye, photophysical parameters, Photo stability, Silicate based sol-gel, Polymeric matrices

Posted Date: June 9th, 2021

DOl: https://doi.org/10.21203/rs.3.rs-577144/v1

License: (c) (i) This work is licensed under a Creative Commons Attribution 4.0 International License.

Read Full License

Version of Record: A version of this preprint was published at Journal of Fluorescence on July 30th, 2021. See the published version at https://doi.org/10.1007/s10895-021-02781-6. 


\title{
Spectral behavior and photophysical parameters of dihydrophenanthro[9,10-e][1,2,4]triazine derivative dyes in sol-gel and methyl methacrylate polymer matrices
}

\author{
Mahmoud E. M. Sakr ${ }^{a *}$, Maram T. H. Abou Kana ${ }^{a}$. Ahmed H.M. Elwahy ${ }^{\text {}}$, Samy A. El- \\ Daly $^{\mathrm{c}}$, El-Zeiny M. Ebeid ${ }^{\mathrm{c}, \mathrm{d}}$. \\ ${ }^{a}$ Laser Sciences and Interactions Department, National Institute of Laser-Enhanced Sciences \\ (NILES), Cairo University, Giza, Egypt. \\ ${ }^{b}$ Chemistry Department, Faculty of science, Cairo University, Giza, Egypt. \\ ${ }^{c}$ Chemistry Department, Faculty of Science, Tanta University, Tanta, Egypt. \\ ${ }^{d}$ Misr University for Science and Technology (MUST), 6th of October City, Egypt.
}

\begin{abstract}
This paper deals the optical and photophysical properties of dihydrophenanthro [9,10-e] $[1,2,4]$ triazine fluorescent derivative dyes doped in Silicate based sol-gel, homo-poly methyl methacrylate (PMMA). Solid hosts effect on the optical and photophysical parameters such as molar absorptivity, cross sections of singlet-singlet electronic absorption and emission spectra, excited state lifetime, quantum yield of fluorescence. Also the dipole moment of electronic transition, the length of attenuation and Oscillator strength of electronic transition from So $\rightarrow \mathrm{S}_{1}$ have been detected. The dyes with different pumping power were pumped by $3^{\text {rd }}$ harmonic $\mathrm{Nd}$ :YAG pulsed laser $8 \mathrm{~ns}$ pulse duration, with a repetition rate at $(10 \mathrm{~Hz})$. Good Photo stability of compound 1 and 2 were obtained that was decreased to $49 \%, 54 \%$ and $46 \%, 40 \%$ of the initial ASE of dyes in sol gel and PMMA respectively, after 55000 pumping pulses at $(10 \mathrm{~mJ} / \mathrm{pulse})$. The dyes in sol-gel show improved Photo stability compared with those in organic polymeric matrices.
\end{abstract}

\footnotetext{
${ }^{*}$ Corresponding author: Mahmoud sakr

E-mail: $\underline{\text { m.sakr@niles.edu.eg }}$
} 
Keywords: Optical property; Solid state laser dye; photophysical parameters; Photo stability; Silicate based sol-gel; Polymeric matrices.

\section{Introduction}

In recent years, the synthesis of new high-performance dyes and the implementation of new ways of incorporating the organic molecules into the solid matrix have resulted in significant advances towards the development of practical tunable solid-state dye lasers[1-3], due to their high efficiency and they do not contain volatile solvents, they are nonflammable, nontoxic, compact in size and mechanically and thermally more stable [4]. In using liquid dye lasers, there are several disadvantages like poor thermal stability of dye solutions, requirement of large volume of solutions and need of significant maintenance, as well as high rate of non-radiative processes due to the high flexibility of dye in solution.[5-8]. Overcome these difficulties by doping dye molecules into restricted host matrices like polymers, silica gels, and xerogels and sol-gel glasses [8, 9]. The solgel method is a method for organic dye molecules incorporated into an inorganic silica host [10]. Which it shows some advantages, e.g. compactness, better manageability and highly porous, transparent in Uv visible-near IR regions. Its reaction can be controlled easily by chemical methods. It allows introducing permanent organic groups to form inorganic-organic hybrid materials [11] and the process takes place at low temperature [12]. In the present study discussed the novelty of fluorescent derivative dye in far UVVis range have been synthesized before by bis-alkylation of starting compound 1, 12adihydrophenanthro $[9,10-\mathrm{e}][1,2,4]$ triazine-3(4H)-thione, with the appropriate dibromo compound in various solid hosts matrices (sol-gel and PMMA) and the effect on the various properties including photo stability, optical and photo- physical properties of dihydrophenanthro $[9,10-\mathrm{e}][1,2,4]$ triazine derivatives doped in sol-gel and methylmethacrylate polymer matrices. Photophysical properties provides basic information about the lasing behavior of the material. The absorption and emission properties of the dyes in sol-gel and PMMA polymer host matrices have been measured. The results are compared with their respective properties in sol-gel and PMMA host matrices. 


\section{Experimental}

\subsection{Materials}

Spectroscopic grade solvents and all chemicals were obtained from Sigma Aldrich and used without further purification. Active chromophore was recently prepared and reported in described in reference [13]

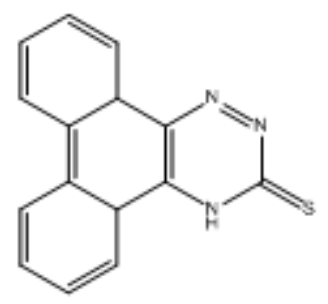

Compound 1,

\section{2a-dihydrophenanthro $[9,10-e][1,2,4]$ triazine-3(4H)-thione}

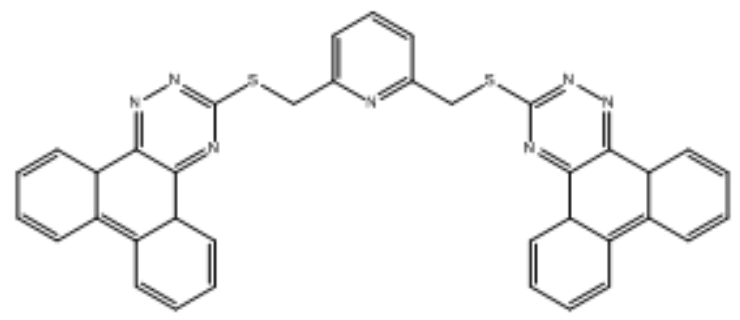

Compound 2,

\section{2,6-Bis $(((4 b, 12 a-d i h y d r o p h e n a n t h r o[9,10-e][1,2,4]$ triazin-3-yl)thio)methyl $)$ pyridine}

Silicate Sol-gel matrix and polymer (using methyl methacrylate MMA as monomer) were prepared as described in references [14] and [15, 16] respectively.<smiles>C=C(C)C(=O)OC</smiles>

Methyl methacrylate (MMA)

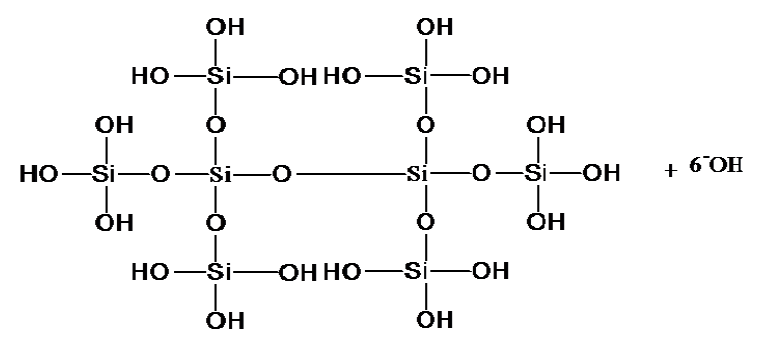

Sol-gel matrix 


\subsection{Spectra measurements}

Dyes samples were doped in transparent sol-gel and PMMA of dye concentrations ranging from $2 \times 10^{-6} \mathrm{M}$ to $2 \times 10^{-4} \mathrm{M}$ in sol gel and ranging from $4 \times 10^{-5} \mathrm{M}$ to $2 \times 10^{-4} \mathrm{M}$ in PMMA. The electronic absorption and fluorescence properties of these dyes in sol-gel and PMMA polymer matrices were studies using a Camspec M501 UV/Vis spectrophotometer and a PF-6300 spectrofluorometer respectively. The wavelengths of excitation were adjusted at absorption maximum wavelengths for each fluorescence measurement of a dye samples. Laser induced fluorescence of dye samples as function of different input pumping energies were carried out by $3^{\text {rd }}$ harmonic Nd:YAG laser using homemade setup reported in [17].The photostability of the dye was also determined using the same experimental setup [17]. The input energy was kept constant at (10 mJ) by pumping with $355 \mathrm{~nm}$ of $3^{\text {rd }}$ harmonic Nd:YAG laser.

\section{3. photophysical parameters calculations:}

Depending upon these absorption and emission spectra, important and significant photophysical parameters could be determined according to the standard equations: The oscillator strength values were calculated from the equation (1) [18].

$$
f=4.32 \times 10^{-9} \int \varepsilon(\mathcal{G}) d \mathcal{G}_{(1)}
$$

Where the wave number is $v$ expressed in $\mathrm{cm}^{-1}$ and $\varepsilon$ is the molar absorptivity $\left(1 \mathrm{~mol}^{-1}\right.$ $\left.\mathrm{cm}^{-1}\right)$. Scientifically the value of $(f)$ is dependent on the molecular structure and the type of electronic transition. The attenuation length $\Lambda(\lambda)$ (the distance at which the original light intensity $\mathrm{I}_{0}$ is reduced to $\mathrm{I}=\mathrm{I}_{0} / \mathrm{e}$, is given by equation (2) [19].

$$
\Lambda(\lambda)=\frac{1}{\varepsilon(\lambda) c \ln (10)},
$$

Where the molar absorptivity is $\varepsilon(\lambda)$ and $\mathrm{c}$ is the concentration. The $\Lambda$ values are mainly dependent on the wavelength. The dipole moment transition $\mu_{12}$ was calculated according to equation (3) [19]. 
$\mu_{12}^{2}=\frac{f}{4.72 \times 10^{-7} E_{\max }},{ }_{(3)}$

Where $\mathrm{E}_{\max }$ is the maximum absorption energy in $\mathrm{cm}^{-1}$. The rate of radiative decay constant $\left(\mathrm{k}_{\mathrm{r}}\right)$ of a fluorophore can be theoretically calculated from equation (4)[20].

$k_{r}=\frac{1}{\tau_{0}}=2.88 \times 10^{-9} n^{2} \frac{\int F(1 \% d v}{\int F\left(1 \% \% \% \%^{3} d v\right.} \int \frac{\varepsilon(1 \%)}{1 \%} d \% \%$ (4)

Where $F(\$ \%), \mathscr{1}, \varepsilon(\$ \%$ are the intensity of fluorescence, the wave number and the molar absorptivity at wave number $(1 /), \mathrm{n}$ is the index of refraction of solid host. The absorption cross-section $\sigma_{\mathrm{a}}$ is given by equation (5)[21].

$$
\sigma_{a}=0.385 \times 10^{-20} \varepsilon(\lambda) \text {.(5) }
$$

The quantum yield of a compound relative to a standard probe, the following relationship (6) was applied: [22, 23].

$\phi_{u}=\phi_{s} \times \frac{I_{u}}{I_{s}} \times \frac{A_{s}}{A_{u}} \times \frac{n_{u}^{2}}{n_{s}^{2}}$.

Where $\phi_{u}$ and $\phi_{s}$ are the fluorescence quantum yields of the unknown and the standard respectively. $I_{u}$ and $I_{s}$ are the areas under emission curves for the unknown and the standard respectively. $A_{u}$ and $A_{s}$ are the absorbance of the unknown, and the standard respectively. $\mathrm{n}_{\mathrm{s}}$ and $\mathrm{n}_{\mathrm{u}}$ are the refractive indices of solvents used. In determination of fluorescence quantum yields $\varphi_{(\mathrm{s})}$ (determined relative to the fluorescein laser dye in ethanol as reference) care was taken to keep the concentration of all the samples at levels low enough to avoid re-absorption of the emitted photons[22, 23]. Fluorescence lifetimes $\left(\tau_{f}\right)$ were calculated by using equation $(7)$.

$$
\tau_{f}=\tau_{0} \phi_{f},(7)
$$

Where $\left(\tau_{0}\right)$ is the natural lifetime $\left(\tau_{0}=1 / \mathrm{k}_{\mathrm{r}}\right)$. The rate constant of intersystem crossing $\left(\mathrm{k}_{\mathrm{isc}}\right)$ is related to the quantum yield $\phi_{\mathrm{f}}$ for $\left(\phi_{\mathrm{f}} \approx 1\right)$ by the relationship (8) [24]: 
$\mathrm{k}_{\mathrm{isc}}=\left(1-\phi_{\mathrm{f}}\right) / \tau_{\mathrm{f}}$.

Where $\phi_{\mathrm{f}}$ is the quantum yield, $\lambda_{\mathrm{A}}$ and $\lambda_{\mathrm{f}}$ are the maximum absorption and fluorescence wavelengths respectively. The emission cross-section $\sigma_{\mathrm{e}}$ was calculated according to equation (9) [25].

$\sigma_{e}=\frac{\lambda^{4} F(\lambda) \phi_{f}}{8 \pi c n^{2} \tau_{f}}$,

Where $\lambda_{e}$ is the wavelength emission, $\mathrm{n}$ is the index of refraction of the host, $\varepsilon$ is the extinction coefficient, $c$ is the light velocity, $F(\lambda)$ is the normalized emission spectrum where $\int \mathrm{F}(\lambda) \mathrm{d} \lambda=1$ and $\phi_{f}$ is the quantum yield.

\section{Results and discussion:}

\subsection{Photophysical parameters in different matrices}

Figure 1 and figure 2 show the UV-vis absorption and fluorescence spectra of the dyes (Cpd 1 and Cpd 2) in sol-gel and homo polymer PMMA polymer matrices. There is a minimum overlap between the dyes absorption and emission spectra in the two solid matrices. This is important as far as reabsorption of emitted photons is concerned.
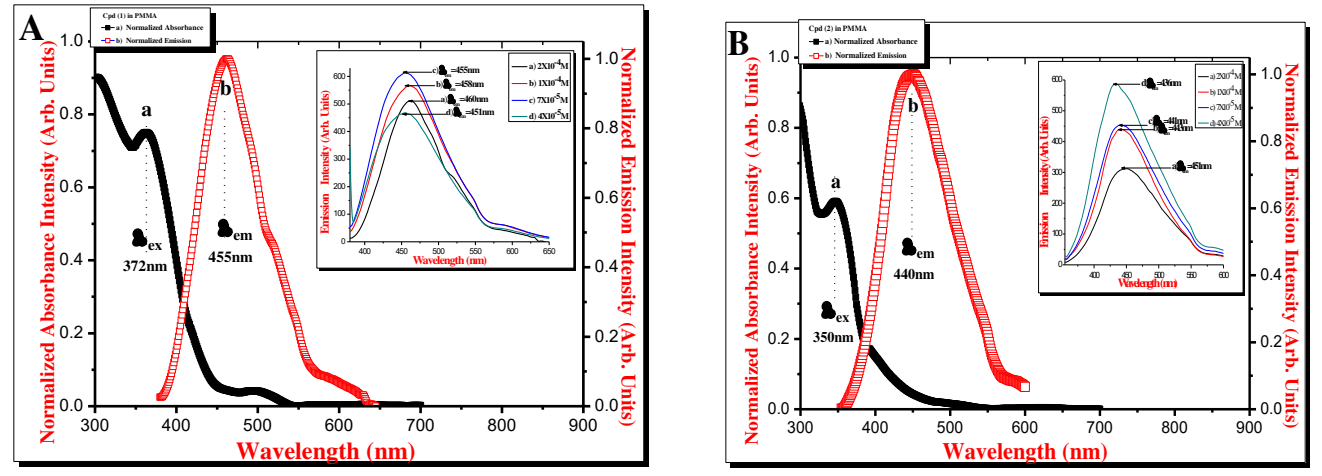

Figure 1 (A, B): Normalized absorbance and emission intensity of A) compound 1 and $B$ ) compound 2 in PMMA respectively. Inset figure : Emission of compounds 1 and 2of different concentrations in PMMA at excitation wavelength A) 372 and B) 350nm respectively. 
Figure 1 (A, B)shows that the absorption maximum peaks were 372 and $350 \mathrm{~nm}$ of compounds 1 and 2 in PMMA respectively. Also, shows that the emission maximum peaks were 455 and $440 \mathrm{~nm}$ of compounds 1 and 2 in PMMA respectively. Inset Figure 1 (A, B)shows that the emission of compounds 1 and 2 of different concentrations in PMMA at excitation wavelength 372 and 350nm respectively. It showed a red shift as the concentration increased represented that in inset figure (1A, 1B). The optimum dye concentration was $7 \times 10^{-5} \mathrm{M}$ and $4 \times 10^{-5} \mathrm{M}$ in PMMA of Cpd 1 and Cpd 2 respectively.
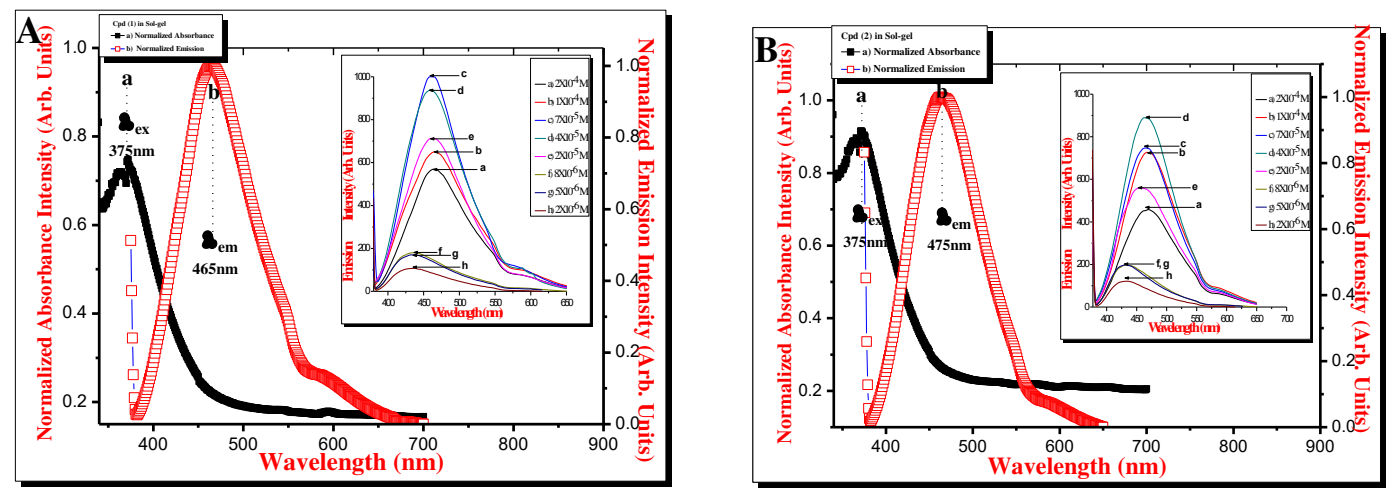

Figure 2 (A, B): Normalized absorbance and emission intensity of A) compound 1and B) compound 2 in sol-gel respectively. Inset figure : Emission of A) compound1 and $B$ ) compound 2 of different concentrations in sol-gel at excitation wavelength 375nmrespectively.

Figure 2 (A, B)shows that the absorption maximum peaks were 375 of compounds 1 and 2 in sol-gel host. Also, shows that the emission maximum peaks were 465 and $475 \mathrm{~nm}$ of compounds 1 and 2 in sol-gel host respectively. Inset Figure 2 (A, B)shows that the emission of compounds 1 and 2of different concentrations in sol-gel host at excitation wavelength 375 respectively. The dye emission spectra with different concentrations doped in sol-gel host was shown in inset figure 2 (A, B). The dye fluorescence peak wavelength showed a large red shift as the concentration increased and the dye fluorescence peak intensity increased till $7 \times 10^{-5} \mathrm{M}$ in case of $\mathrm{Cpd} 1$ and $4 \times 10^{-5}$ in case of Cpd2 then decreased with increasing concentrations which might be attributed to due molecular aggregations of dye molecules. Also the optimum dye concentration was $7 \times 10^{-}$ ${ }^{5} \mathrm{M}$ and $4 \times 10^{-5} \mathrm{M}$ in sol-gel of $\mathrm{Cpd} 1$ and Cpd 2 respectively. Comparative the dye fluorescence peak wavelengths and intensities in different solid matrices showed that, the 
fluorescence emission wavelengths are higher red shifted in sol-gel compared to polymer matrices. This behavior indicates a more relaxed, excited state due to dyes host interaction within excited state lifetime. Some important photo-physical parameters of the dye were calculated and summarized in the table (1), which proved their future applicable action in advanced optical applications. However, the absorption cross-section $\left(\boldsymbol{\sigma}_{\mathbf{a}}\right)$ is the ability of the molecule to absorb a photon of a certain polarization and wavelength. Emission cross-section ( $\boldsymbol{\sigma e})$ measures the rate of optical transitions (per active ion) starting from a certain electronic level time the photon flow. The attenuation length (L) (called absorption length) is the distance into a material when the probability has dropped to1/e that a particle has not been absorbed.

Table 1: photo-physical parameters of $7 \times 10^{-5} \mathrm{M}$ and $4 \times 10^{-5} \mathrm{M}$ of $\mathrm{Cpd} 1$ and $\mathrm{Cpd} 2$ in

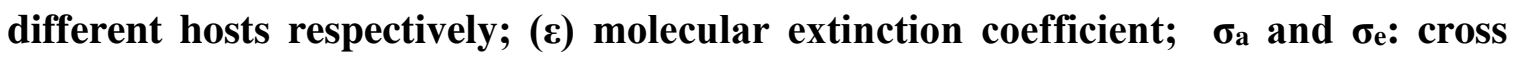
section of absorption and emission; $(\Lambda)$ the attenuation length, $\left(\tau_{f}\right)$ fluorescence life time, $\left(\tau_{0}\right)$ calculated fluorescence life time, $\mu_{12}(D)$ the dipole moment transition, $\left(E_{f}\right)$ energy yield of fluorescence, $\left(K_{r}\right)$ the radiative decay rate , $\left(K_{\text {isc }}\right)$ the rate of intersystem crossing, (f) oscillator strength, $\varphi$ f fluorescence quantum yield, indifferent hosts:

\begin{tabular}{|c|c|c|c|c|c|c|c|c|c|c|c|c|c|}
\hline \multirow{2}{*}{\multicolumn{2}{|c|}{ Sample /solvent }} & & $\sigma_{a}$ & $\sigma_{e}$ & & & & & & $\mathbf{K}_{\mathbf{r}}$ & $\mathbf{K}_{\text {isc }}$ & & \\
\hline & & $\begin{array}{l}{ }^{1} \cdot \mathbf{C m}^{-1} \\
\left(\mathbf{1 0}^{4}\right)\end{array}$ & $\begin{array}{l}\left.{ }^{16}\right) \\
\mathbf{C m}^{2}\end{array}$ & $\begin{array}{c}\left.{ }^{17}\right) \\
\mathbf{C m}^{2}\end{array}$ & & (ns) & (ns) & (D) & & $\mathrm{s}^{-1}$ & $\mathrm{~s}^{-1}$ & & \\
\hline \multirow{2}{*}{ Cpd1 } & Sol-gel & 8.52 & 3.2 & 1,94 & 0.05 & 0.32 & 0.52 & 6.53 & 0.49 & $\mathbf{1 . 9 2}$ & 1.20 & 0.65 & 0.62 \\
\hline & PMMA & 11.52 & 4.4 & 2.45 & 0.03 & 0.28 & 0.42 & 8.35 & 0.56 & 2.40 & 1.14 & 0.75 & 0.68 \\
\hline \multirow{2}{*}{ Cpd2 } & Sol-gel & 10.91 & 4.8 & 2.35 & 0.039 & 0.31 & 0.45 & 8.94 & 0.55 & 2.22 & 0.96 & 0.85 & 0.70 \\
\hline & PMMA & 9.58 & 3.6 & 1.84 & 0.045 & 0.29 & 0.48 & 7.96 & 0.47 & 2.08 & 1.40 & 0.62 & 0.60 \\
\hline
\end{tabular}

It noticed from photo-physical parameters of the dyes in different solid hosts that the intersystem crossing rate constants $\left(\mathrm{k}_{\mathrm{isc}}\right)$ values for compound 1 in sol-gel glass is higher 
compared with polymer matrices and for compound 2 in sol-gel is a lower compared with polymer matrices. This indicates a dynamic quenching process in which the polymer matrices interact with the excited state for compound 2 dye molecules. The oscillator strength value in polymer matrices is higher than those in sol-gel matrix for a compound 1 and in sol-gel matrix is higher than those in polymer matrices for a compound 2 . Hence, the effective number of electrons transferred from the ground to excited states in polymer matrices is higher than those in sol-gel matrix for a compound 1 and in sol-gel is higher than in polymer matrices for a compound 2. Fluorescence quantum yield $\left(\phi_{\mathrm{f}}\right)$ values are lower in sol- gel compared with those in polymer for a compound 1 and are lower in polymer compared with those in sol- gel for a compound 2, indicating more interaction between dye molecules and polymer matrices for a compound 2 . The carbonyl group in chromophore polymer matrices possesses $\left(n, \pi^{*}\right)$ electronic states that are characterized by low singlet - triplet splitting energies $\left(\Delta \mathrm{E}_{\mathrm{S}, \mathrm{T}}\right)$ leading to triplet state population, with subsequent fluorescence quenching [19]. It is known that the $\left(T_{1} \rightarrow T_{n}\right)$ transition is a spin- allowed one that can quench fluorescence by photon reabsorption. The lower energy level of $\left(n, \pi^{*}\right)$ states also allows for exciton trapping [19] adding to factors causing fluorescence quenching. However, for the compound 2 only noted that the role of polymer matrices in fluorescence quenching comes from the higher intersystem crossing rate constants $\left(\mathrm{k}_{\mathrm{isc}}\right)$ values in polymer matrices compared with sol-gel glass.

\subsection{Laser-induced fluorescence of dihydrophenanthro [9, 10- $e][1,2,4]$ triazine derivative:}

The dihydrophenanthro[9,10-e][1,2,4]triazine fluorescent derivative dyes in PMMA polymer and sol-gel were pumped by $3^{\text {rd }}$ harmonic Nd:YAG $(\lambda=355 \mathrm{~nm})$. The spontaneous fluorescence intensities various with wavelength in different hosts as PMMA and sol-gel of different dyes compound 1 and 2 pumped by $3^{\text {rd }}$ harmonic $\mathrm{Nd}$ :YAG pulsed laser at different input power 5, 10 and $20 \mathrm{~mJ}$ shown in figures 3 and 4 $(\mathrm{A}, \mathrm{B})$. 

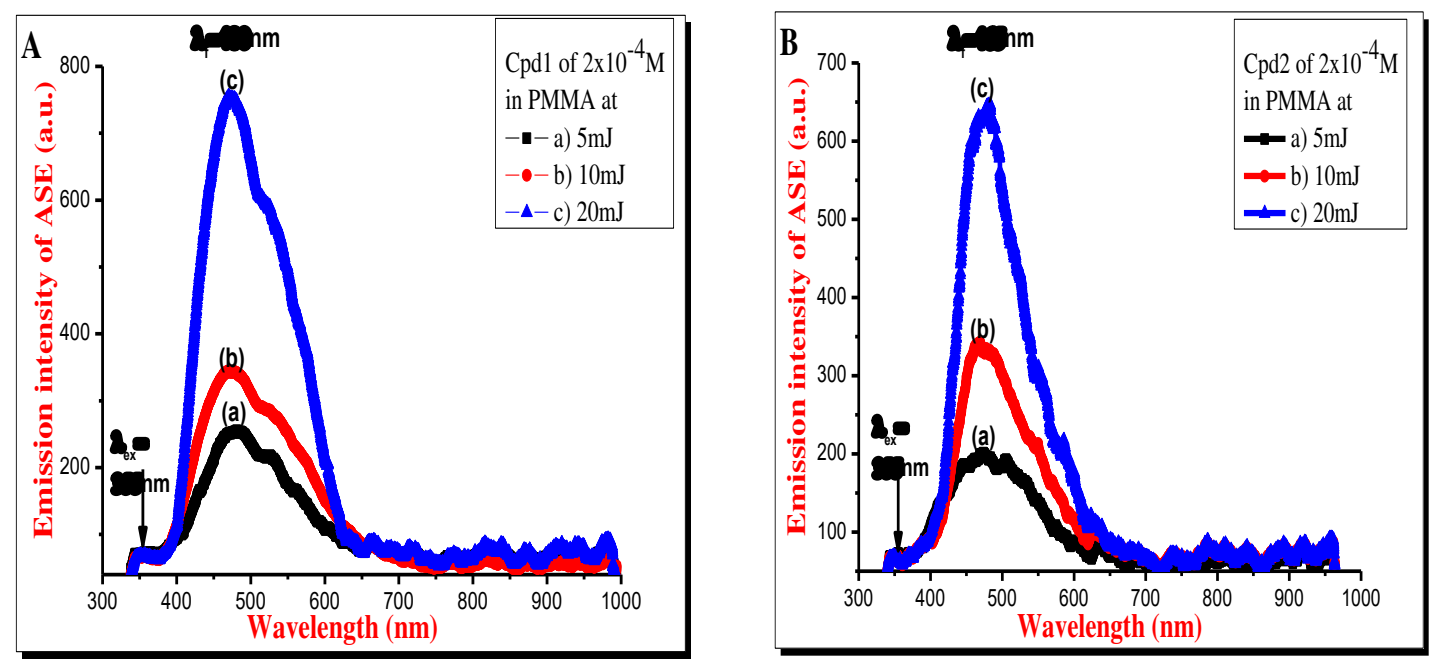

Figure 3 (A, B): Emission intensities of ASE of A) compound 1, B) compound 2, in PMMA, with excitation wavelength $\lambda_{\mathrm{ex}}=355 \mathrm{~nm}$ by $3^{\text {rd }}$ harmonic Nd:YAG pulsed laser at different input pumping power $5 \mathrm{~mJ}, 10 \mathrm{~mJ}, 20 \mathrm{~mJ}$.
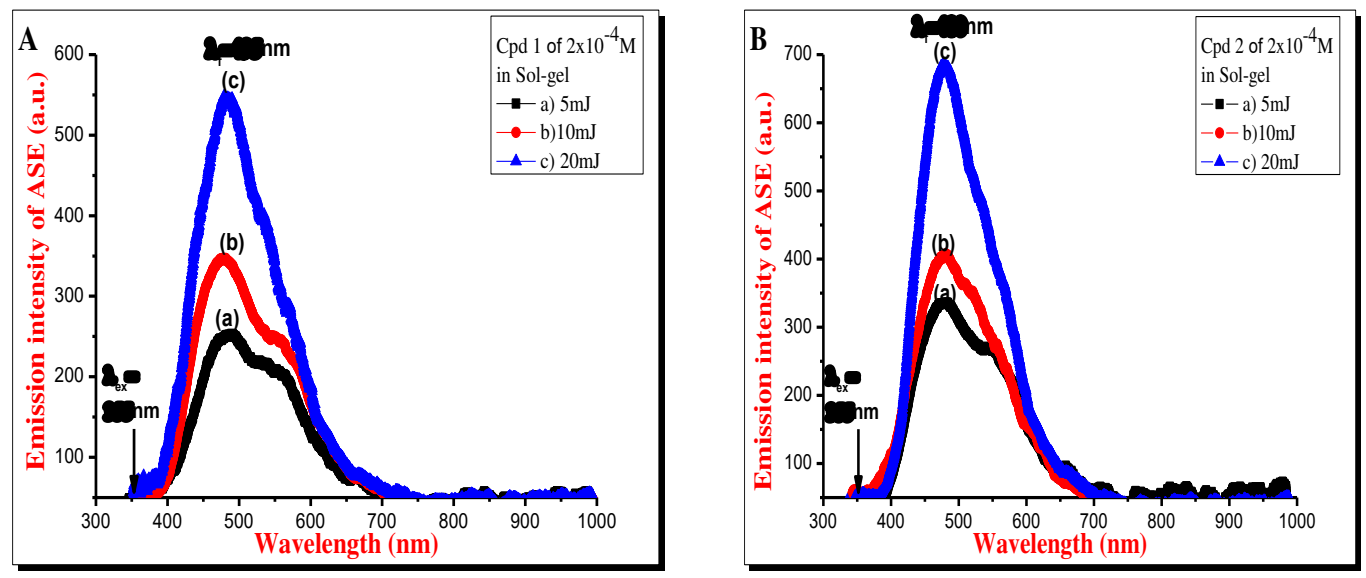

Figure 4 (A, B): Emission intensities of ASE of A) compound 1, B) compound 2 in sol-gel, with excitation wavelength $355 \mathrm{~nm}$ by $3^{\text {rd }}$ harmonic Nd:YAG pulsed laser at different input pumping power $5 \mathrm{~mJ}, 10 \mathrm{~mJ}, 20 \mathrm{~mJ}$.

By pumping using $355 \mathrm{~nm} 3^{\text {rd }}$ harmonic $\mathrm{Nd}$ :YAG pulsed laser with $\lambda_{\mathrm{ex}}=355 \mathrm{~nm}$ excitation wavelength the emission intensity of ASE peak wavelength of two different dyes compound 1 and 2 in sol-gel matrix $\left(\lambda_{\mathrm{f}}=495\right.$ and $\left.\lambda_{\mathrm{f}}=480 \mathrm{~nm}\right)$ respectively showed a large 
red shift from that of the dyes compound 1 and 2 in PMMA $\left(\lambda_{\mathrm{f}}=480\right.$ and $\left.\lambda_{\mathrm{f}}=465 \mathrm{~nm}\right)$ in figures 3 and 4 (A, B). The figures 3 and 4 (A, B) showed the emission intensity of the two dyes in solid hosts at different input power at 5, 10 and $20 \mathrm{~mJ}$. The increasing of the peak intensity of ASE of the dye may be attributed to the increasing of the number of excited molecules (increase the population of $\mathrm{S}_{1}$ state) which yields more emitted photons. These changes in wavelength due to the interaction of the structure of the two different dyes molecules with different solid matrices as shown in figures 3 and 4 (A, B). The photostability as important photochemical parameter was studied by the evolution of the output fluorescence various with number of pulses, in the same position of the samples, as in figure 5 (A, B). This study were carried out for the samples of the dyes which the repetition rate of $355 \mathrm{~nm} \mathrm{Nd}: \mathrm{YAG}$ laser with $8 \mathrm{~ns}$ pulse duration was kept at $(10 \mathrm{~Hz})$ and the energy was kept constant at $(10 \mathrm{~mJ} / \mathrm{pulse})$. The output energy gradually decreased due to the photodegradation progressive and thermo-degradation of the dyes molecules. This decreasing occurred at a faster rate for the dyes in polymer than sol gel, and the peak ASE dropped to $46 \%, 40 \%$ and $49 \%, 54 \%$ of the initial ASE of the dyes (Cpd1 and Cpd2) in PMMA and sol-gel respectively by pumping with $355 \mathrm{~nm} 3^{\text {rd }}$ harmonic Nd:YAG laser at $10 \mathrm{~mJ}$. Since the mechanism of photodegradation occurs by the interaction molecules of the dye in the excited state with other species such as impurities, other dye molecules and singlet oxygen. Through the process of doping dyes into a solid medium, the photochemical pathways including bimolecular reactions can be suppressed by caging or trapping the dye within a solid host [26].
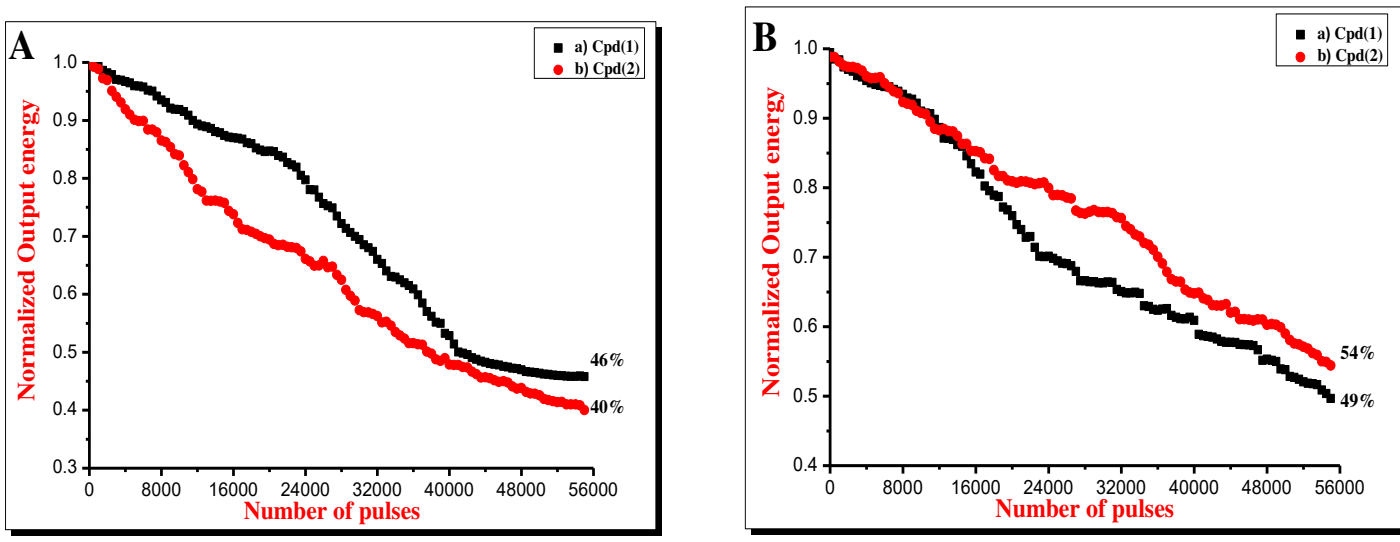

Figure 5(A, B): Normalized output energy of the dyes compound 1 and 2 in A) 


\section{PMMA and B) Sol-gel as a function of the number of pump pulses using pumping power of $355 \mathrm{~nm} 3^{\text {rd }}$ harmonic Nd:YAG laser at $10 \mathrm{~mJ}$}

The improved photostability of the dyes within solid hosts has been attributed for caging and molecules immobilizing of the dyes, minimizing thereby excited-state interaction with other species including molecular oxygen. So, the dye photodegradation in a solid host depends on the dyes molecule nature, the host composition and structure, and the host impurities as well as presence of molecular oxygen. Also, the improvement of the photostability and decrease the dyes photodegradation, the surroundings of the dyes molecules must be controlled. The major silicate matrices advantages compared with organic polymeric ones. e.g. thermally stability much beyond the range of temperatures which are of relevance for polymeric matrices, they do not photo degrade and, also, are not involved in light induced reactions with the matrix. Another possible factor that may explains the reduced rate of degradation in the more rigid matrices may be that the dyes molecules are more tightly confined within the pores of the matrix, limiting rotational and translational freedom. A mobile molecule, as in solution, will be more likely to encounter an oxygen molecule and undergo degradation. Less freedom, as defined by the restrictive pores of the matrix, may make the dyes molecule less likely to interact with molecular oxygen leading to photodegradation or molecular oxygen fluorescence quenching. is a well-known fluorescence quencher due to its paramagnetic nature which enhances the rate of intersystem crossing[26].

\section{Conclusion:}

The optical absorption and emission properties of the dyes have been studied in different solid hosts such as sol-gel and PMMA polymer. Their respective spectroscopic and photophysical parameters meet the best requirements for a good laser dye such as high molar absorption coefficients at the wavelength of the pump laser, broad spectral region of fluorescence and high fluorescence quantum yield, short fluorescence decay time, large Stokes' shift, little overlap of the fluorescence and triplet absorption spectral regions, photochemical stability. Pumping the samples using $3^{\text {rd }}$ harmonic Nd:YAG laser 
$(\lambda=355 \mathrm{~nm}$ ) showed different emission wavelength of ASE peak. It were nearly in PMMA polymer $\left(\lambda_{\mathrm{f}}=480 \mathrm{~nm}\right),(\lambda \mathrm{f}=465 \mathrm{~nm})$ and in sol-gel matrix $\left(\lambda_{\mathrm{f}}=495 \mathrm{~nm}\right),\left(\lambda_{\mathrm{f}}=480 \mathrm{~nm}\right)$ of compound 1 and 2 respectively. The nature of solid host has significant effect on spectroscopic properties of dye. Good dye photostability were obtained that was decreased to 46\%, 40\% and 49\%, 54\% of the initial ASE of the dyes (Cpd1 and Cpd2) in

PMMA and sol-gel respectively of pumping with $355 \mathrm{~nm} 3^{\text {rd }}$ harmonic Nd:YAG laser $8 \mathrm{~ns}$ pulse duration, with a repetition rate at $(10 \mathrm{~Hz})$ and the energy was kept constant at $(10$ $\mathrm{mJ} /$ pulse). after 55000 pulses.

\section{Declaration of Competing Interest}

The authors declare that they have no known competing financial interests or personal relationships that could have appeared to influence the work reported in this paper.

\section{Funding}

No funding

\section{Ethics approvals}

Done

\section{Consent to participate}

Not applicable

Consent for publication

Not applicable

\section{Availability of data and material}

All data generated or analysed during this study are included in this published article

\section{Code availability}

Not applicable

\section{Author Agreement}


All authors have seen and approved the final version of the manuscript being submitted. And warrant that the article is the authors' original work, hasn't received prior publication and isn't under consideration for publication elsewhere.

\section{Credit authorship contribution statement}

Mahmoud E. M. Sakr: methodology, writing - review and editing.

Maram T. H. Abou Kana: conceptualization, investigation, supervision, writing original draft.

Ahmed H.M. Elwahy: Conceptualization, supervision, investigation, synthesis, resources.

Samy A. El-Daly: review, editing and supervision.

El-Zeiny M. Ebeid: Supervision.

\section{Reference:}

[1] R. E. Hermes, T. H. Allik, S. Chandra, and J. A. Hutchinson, "High efficiency pyrromethene doped solid-state dye laser," Appl. Phys. Lett. 63(1993) 877.

[2] B. Dunn, F. Nishida, K. Toda, J. Zin, T. Allik, S. Chandra, and J. A. Hutchinson, "Advances in dye doped sol-gel lasers," in New Materials for Advanced Solid-State Lasers, Mater. Res. Soc. Symp. Proc. 329 (1994) 267.

[3] M. Faloss, M. Canva, P. Georges, A. Brun, F. Chaput, and J. P. Boilot, "Toward millions of laser pulses with pyrromethene and perylene-doped xerogels," Appl. Opt. 36 (1997) 6760.

[4] M. Canva, B. Darracq, F. Chaput, K. Lahlil, F. Bentivegna, M. Brunel, M. Faloss, P. Georges, A. Brun, J. P. Boilot, and Y. Levy, "Functionalized dye-doped hybrid sol-gel materials for solid-state dye laser to nonlinear applications and organic photo reactivity," in Organic-Inorganic Hybrid Materials for Photonics, L. G. Hubert-Pfalzgraf and S. Najafi, eds., Proc. SPIE 3469 (1998) 164.

[5] F. P. Schafer. Dye Lasers. New York: Springer; (1990). 
[6] D. Avnir, D. Levy, R. Reisfeld. The nature of the silica cage as reflected by spectral changes and enhanced photostability of trapped Rhodamine 6G J Phys Chem; 88 (1984) 5956.

[7] D. Avnir, V. R. Kaufman, R. Reisfeld Organic fluorescent dyes trapped in silica and silica-titania thin films by the sol-gel method. Photophysical, film and cage properties $J$ Non-Cryst Solids; 74 (1985)395.

[8] A. Bergmann, W. Holzer, R. Stark, H. Gratz, A. Penzkofer, F. Amat Guerri, A. Costela, I. Garcia Moreno, and R. Sastre, "Photo physical characterization of pyrromethene dyes in solid matrices of acrylic copolymers," Chem. Phys. 271 (2001) 201.

[9] A.V. Deshpande, U. Kumar Effect of method of preparation on photophysical properties of Rh-B impregnated sol-gel hosts J Non-Cryst Solids 306 (2002) 149.

[10] V. A. Deshpande, V. L. Jathar, R. R. Jayraj Effect of Matrix Treatment on Spectroscopic Properties of $\mathrm{HCl}$ Catalysed Sol-Gel Glasses Containing Coumarin Laser Dyes J Fluoresc19 (2009) 607.

[11] L. L. Hench, J. L. Nogues Sol-Gel Processing of Net Shape Silica Optics J Sol-Gel Opt; 295 (1994) 59.

[12] R. Wang, U. Narang, N.P. Paras, F.V. Bright. Affinity of antifluorescein antibodies encapsulated within a transparent sol-gel glass J Anal Chem65 (1993) 2671.

[13] M. E. M. Sakr, M. T. H. Abou Kana, A. H. M .Elwahy, S. A. El-Daly, E. M. Ebeid. Novel far UV-Vis absorbing bis(dihydrophenanthro[9,10-e][1,2,4]triazine) derivative dyes: Synthesis, optical, photophysical and solvatochromic properties, Journal of Molecular Structure 1206 (2020) 127690.

[14] V. A. Deshpande, R. R. Jayraj, V. J. Laxman. Comparison of spectroscopic and lasing properties of different types of sol-gel glass matrices containing Rh-6G $J$ Fluoresc19 (2009) 1083.

[15] M. Macret, G. Hild. Hydroxyalkyl methacrylates: hydrogel formation based on the radical copolymerization of 2-hydroxyethyl methacrylate and 2,3dihydroxypropyl methacrylate $J$ Polym 23 (1982) 748. 
[16] M. Ilavski, K. Dusek, J. Vacik, J. Kopecek. Deformational, swelling, and potentiometric behavior of ionized gels of 2-hydroxyethylmethacrylate-methacrylic acid copolymers J Appl Polym Sci 23 (1979) 2073.

[17] D. M. AL-Aqmar, H. I. Abdelkader, Maram T.H. Abou Kana. Spectroscopic properties and amplified spontaneous emission of fluorescein laser dye in ionic liquids as green media Optical Materials 47 (2015) 573.

[18] V. Bojinov and I. Grabchev, Synthesis and photophysical investigations of novel combined benzo [de]anthracen-7-one/2, 2, 6, 6-tetramethylpiperidines as fluorescent stabilisers for polymer materials J. Polymer degradation and Stability.85 (2004) 789.

[19] E. M. Ebeid and S. M. Alhazmy, Photophysical and Laser-Based Techniques Chemistry, Biology and Medicine, Book Surge Publisher, Charleston, SCUSA. (2006).

[20] G. A. Kumar and N. V. Unnikrishnan, Energy transfer and optical gain studies of FDS: Rh B dye mixture investigated under cw laser excitation J. Photochemistry and Photobiology Chemistry.144 (2001) 107.

[21] S. A. El-Daly, Photophysical properties: laser activity of and energy transfer from 1,4-bis[ $\beta$-(2-benzothiazolyl)vinyl]benzene (BVB) J. Photochem, Photobiol. A: Chem. 124 (1999) 127.

[22] A. V. Deshpande and E. B. Namdas, Efficient lasing action of rhodamine 6G in Nafion membranes J. Chem. Phys. Letter.263 (1996) 449.

[23] S. Kumar, V.C. Rao and R. C. Rastogi, Excited-state dipole moments of some hydroxy coumarin dyes using an efficient solvatochromic method based on the solvent polarity parameter, EnT J. SpectrochimicaActa Part A.57 (2001) 41.

[24] T. G. Pavlopoulos, Scaling of dye lasers with improved laser dyes J. progress in Quantum electronics.26 (2002) 193.

[25] J. Heldt, J. Szezepanski and A. Diehl, Gain and excited singlet and triplet state absorption spectra of some 9-acetoxy-10(halogenoacetoxy)phenylanthracenes J. Appl. Phys. Chem. B 46 (1988) 339.

[26] T. Suratwala , Z. Gardlund, K. Davidson , DR. Uhlmann Photostability of silylated coumarin dyes in polycerams hosts J Sol-Gel Sci Technol 8 (1997) 973 
Figures
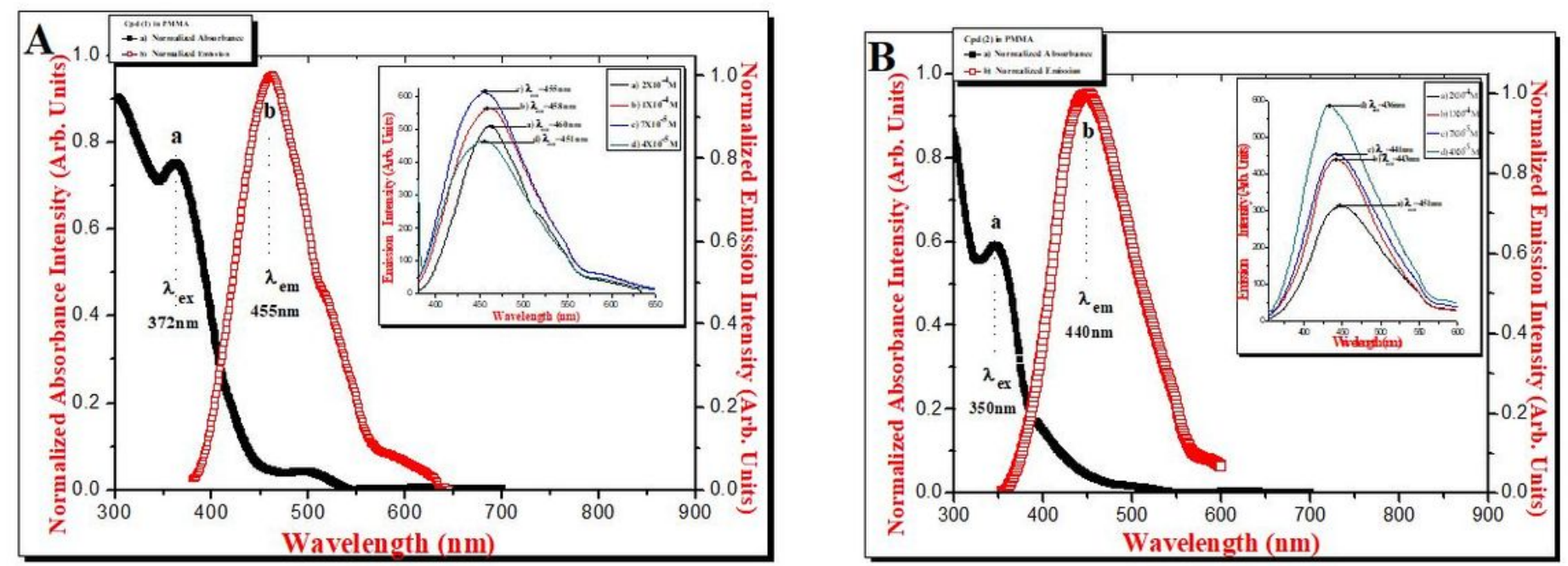

Figure 1

$(A, B)$ : Normalized absorbance and emission intensity of $A$ ) compound 1 and $B$ ) compound 2 in PMMA respectively. Inset figure : Emission of compounds 1 and 2of different concentrations in PMMA at excitation wavelength A) 372 and B) $350 \mathrm{~nm}$ respectively.
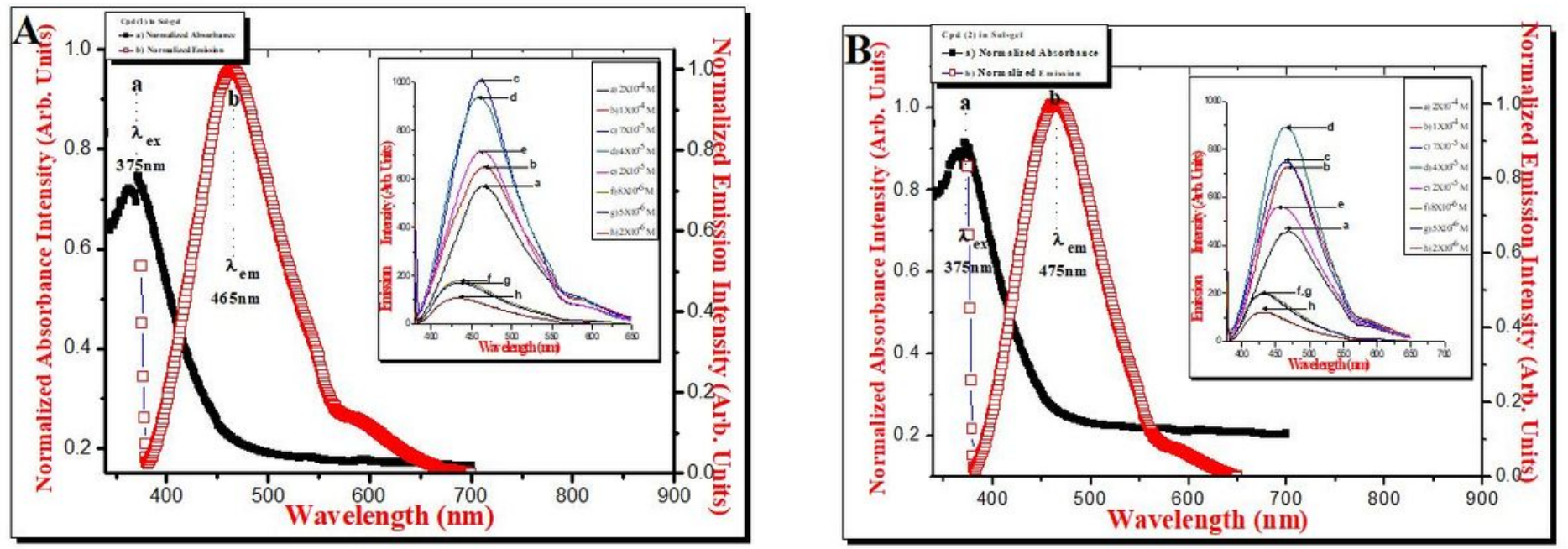

Figure 2

$(A, B)$ : Normalized absorbance and emission intensity of $A$ ) compound 1and B) compound 2 in sol-gel respectively. Inset figure : Emission of A) compound1 and B) compound 2 of different concentrations in sol-gel at excitation wavelength 375nmrespectively. 

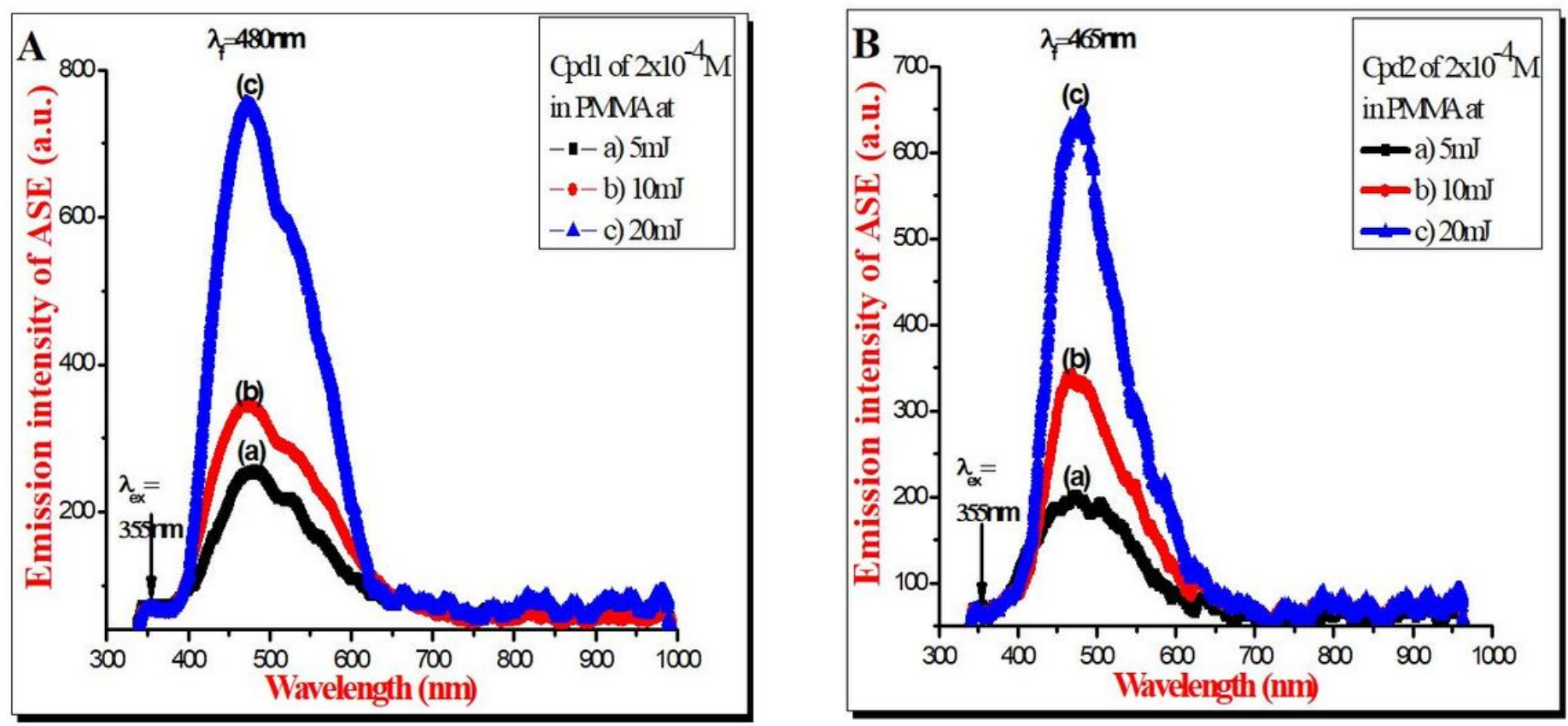

Figure 3

(A, B): Emission intensities of ASE of A) compound 1, B) compound 2, in PMMA, with excitation wavelength $\lambda$ ex $=355 \mathrm{~nm}$ by $3 \mathrm{rd}$ harmonic Nd:YAG pulsed laser at different input pumping power $5 \mathrm{~mJ}$, $10 \mathrm{~mJ}, 20 \mathrm{~mJ}$.
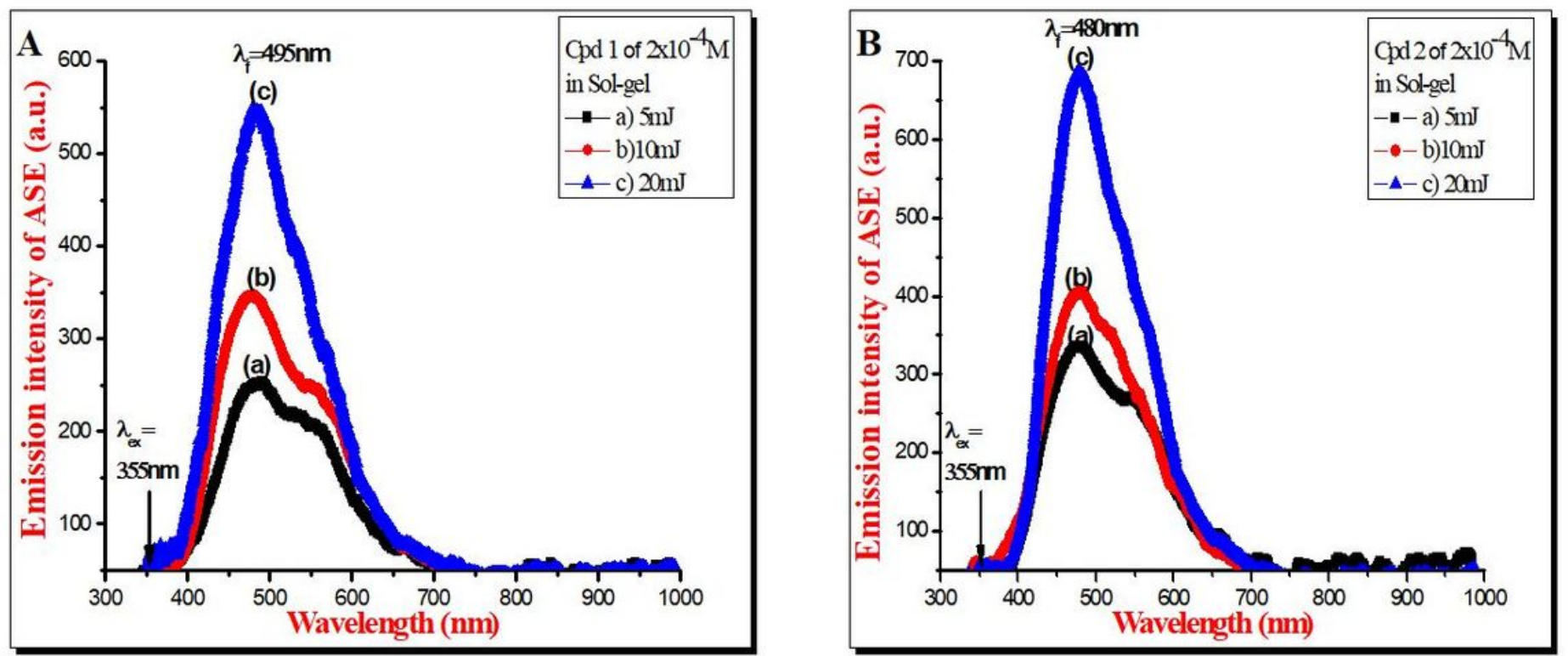

Figure 4

(A, B): Emission intensities of ASE of A) compound 1, B) compound 2 in sol-gel, with excitation wavelength $355 \mathrm{~nm}$ by $3 \mathrm{rd}$ harmonic $\mathrm{Nd}$ :YAG pulsed laser at different input pumping power $5 \mathrm{~mJ}, 10 \mathrm{~mJ}$, $20 \mathrm{~mJ}$. 

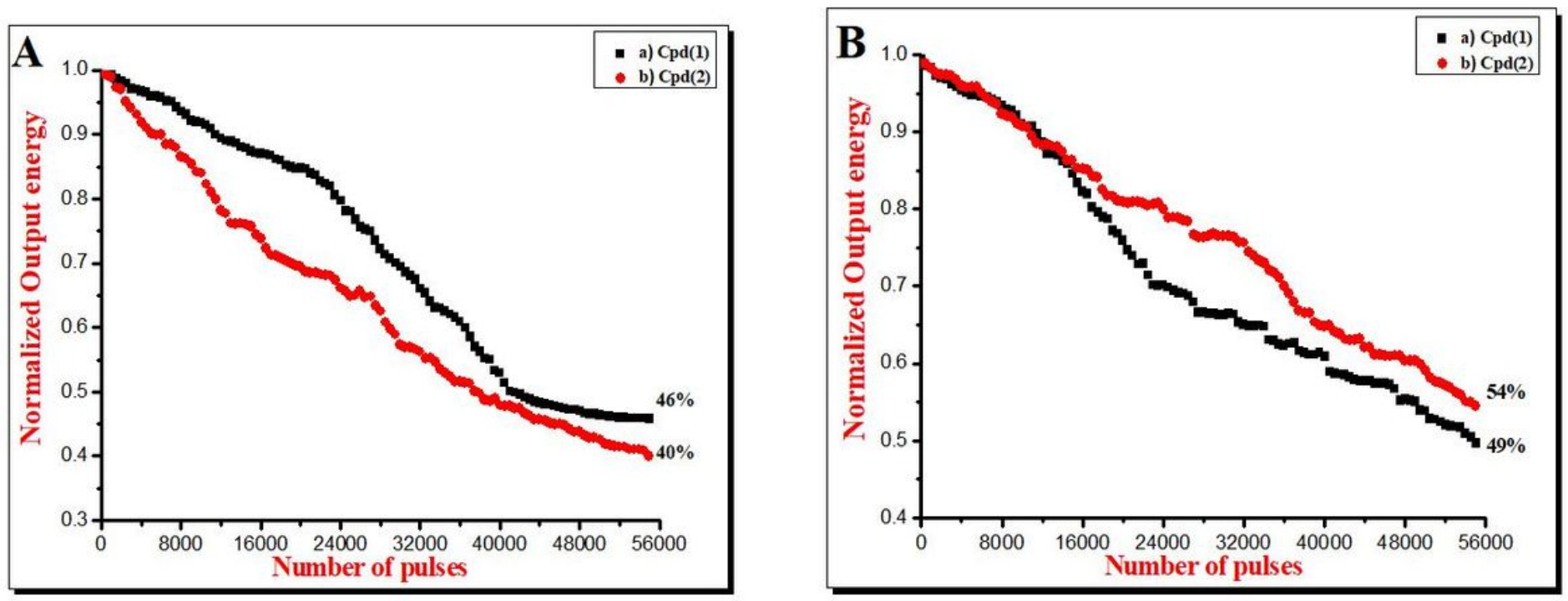

Figure 5

(A, B): Normalized output energy of the dyes compound 1 and 2 in $A$ )

\section{Supplementary Files}

This is a list of supplementary files associated with this preprint. Click to download.

- compound1.jpg

- compound2.jpg

- compound3.jpg 